\title{
CALIDAD DEL CUIDADO FAMILIAR Y SU EFECTO EN EL NEURODESARROLLO DE INFANTES CON VIH/SIDA
}

\author{
QUALITY OF FAMILY CARE AND ITS EFFECT ON THE \\ NEURODEVELOPMENT OF CHILDREN WITH HIV/AIDS
}

\author{
QUALIDADE DO CUIDADO FAMILIAR E SEU EFEITO NO \\ DESENVOLVIMENTO NEUROLÓGICO DE CRIANÇAS COM HIV/AIDS
}

\author{
Nancy Cirila Elliott Rodríguez
}

\begin{abstract}
RESUMEN
Objetivo: Evaluar el efecto de la calidad del cuidado familiar en el neurodesarrollo de infantes de edad entre 3 y 6 ańos infectados con VIH/SIDA. Material y Método: Investigación descriptiva de corte transversal en una muestra de 20 diadas entre cuidadores familiares y sus niños con diagnóstico de VIH/SIDA, hospitalizados en instituciones de salud de la ciudad de Lima metropolitana. El neurodesarrollo fue valorado mediante la Escala Abreviada de Desarrollo 3 (EAD-3) y la calidad del cuidado familiar con el inventario Home Observation for Measurement of the Environment (HOME). Resultados: Entre el 30 y 40\% de los infantes se encuentra en situación de riesgo de retraso del neurodesarrollo en todas las dimensiones evaluadas: motricidad gruesa y fina, audición, lenguaje y el área personal y social; al valorar la calidad del cuidado y su efecto en el neurodesarrollo de los infantes se obtuvo una correlación positiva y significativa $(\mathrm{p}<0,05)$ entre ambas variables; las dimensiones aceptación y materiales de aprendizaje, del inventario $H O M E$ fueron las que más influyen en el neurodesarrollo de los infantes. Conclusiones: La calidad del cuidado familiar hacia el infante con VIH/SIDA ejerce un efecto positivo en el neurodesarrollo de los niños que conformaron la muestra de estudio. La EAD-3 y el inventario $H O M E$ se presentan como herramientas útiles de fácil y rápida aplicación para la valoración oportuna del nivel de neurodesarrollo y calidad de cuidado familiar en infantes.
\end{abstract}

Palabras clave: Cuidado del Niño; Cuidador familiar; Neurodesarrollo; VIH; SIDA.

\begin{abstract}
Objective: To assess the effect of the quality of family care on the neurodevelopment of children aged 3-6 years infected with HIV/AIDS. Materials and Methods: Descriptive cross-sectional research in a sample of 20 dyads between family caregivers and their children diagnosed with HIV/AIDS, hospitalized in health institutions in the city of Lima, Peru. Neurodevelopment was assessed using the Abbreviated Developmental Scale 3 (ADS-3) and the quality of family care with the Home Observation for Measurement of the Environment (HOME) inventory. Results: Between $30 \%$ and $40 \%$ of the children are at risk of neurodevelopmental delay in all the
\end{abstract}

*Licenciada en Enfermería, Magíster en Docencia Universitaria, especialista en Enfermería Pediátrica, Universidad Nacional Federico Villarreal, Lima, Perú. ORCID: https://orcid.org/0000-0003-1136-1250. Email: nancyelliott15@gmail.com 
dimensions evaluated: gross and fine motor skills, hearing, language, and personal and social areas. When assessing the quality of care and its effect on the neurodevelopment of the children, a positive and significant correlation $(\mathrm{p}<0.05)$ was obtained between both variables. The acceptance and learning materials dimensions of the HOME inventory had the greatest influence on the neurodevelopment of the children. Conclusions: The quality of family care for children with HIV/AIDS had a positive effect on the neurodevelopment of the children in the study sample. The ADS-3 and the HOME inventory are useful tools that can be easily and quickly applied for the timely assessment of the level of neurodevelopment and quality of family care in children.

Key words: Child Care; Family Caregiver; Neurodevelopment; HIV; AIDS.

\section{RESUMO}

Objetivo: Avaliar o efeito da qualidade do cuidado familiar no desenvolvimento neurológico de crianças de 3-6 anos de idade infectadas com HIV/AIDS. Material e Método: Pesquisa transversal descritiva em uma amostra de 20 díades entre cuidadores familiares e seus filhos diagnosticados com HIV/AIDS, internados em instituições de saúde da cidade de Lima. O neurodesenvolvimento foi avaliado utilizando a Escala de Desenvolvimento Abreviada 3 (EDA-3) e a qualidade do cuidado familiar com o inventário Home Observation for Measurement of the Environment (HOME). Resultados: Entre 30\% e 40\% das crianças estão em risco de atraso no desenvolvimento neurológico em todas as dimensóes avaliadas: habilidades motoras brutas e finas, audição, linguagem e áreas pessoais e sociais. Ao avaliar a qualidade dos cuidados e seu efeito sobre o neurodesenvolvimento das crianças, foi obtida uma correlação positiva e significativa $(p<0,05)$ entre as duas variáveis. As dimensôes de aceitação e materiais de aprendizagem do inventário HOME foram as mais influentes no desenvolvimento neurológico das crianças. Conclusôes: A qualidade dos cuidados familiares para crianças com HIV/AIDS tem um efeito positivo no desenvolvimento neurológico das crianças da amostra do estudo. O EDA-3 e o inventário HOME são apresentados como ferramentas úteis de aplicação fácil e rápida para a avaliação oportuna do nível de neurodesenvolvimento e da qualidade do cuidado familiar em crianças.

Palavras-chave: Cuidado da criança; Cuidador Familiar; Neurodesenvolvimento; HIV; AIDS.

Fecha de recepción: 08/06/2020

Fecha de aceptación: 29/03/2021

\section{INTRODUCCIÓN}

En la actualidad, son muchos los infantes que presentan, en su corta vida, el desafío de luchar contra condiciones desfavorables para alcanzar su máximo potencial, siendo los más afectados los provenientes de países de mediano y bajos ingresos, donde los niveles de pobreza agudizan su situación $y$ afectan de manera negativa el desarrollo cognitivo y emocional del infante; esto debido, en muchos casos, a la limitada estimulación a nivel cognitivo o por una nutrición deficiente ${ }^{(1-4)}$.

De acuerdo a la UNICEF, los niños y adolescentes son los más afectados por el virus de la inmunodeficiencia humana (VIH) y, por consiguiente, del padecimiento del síndrome de inmunodeficiencia adquirida (SIDA). Se estima que, a nivel mundial, alrededor de casi 1 millón de nacimientos de bebés están expuestos al VIH, cifra que se eleva con las estadísticas relacionadas con aquellos que se infectan durante la lactancia. Esta circunstancia, se ve agravada cuando buena parte de esta población tiene escaso acceso a pruebas y tratamientos que mejorarían su calidad de vida, e incluso, salvarla ${ }^{(5,6)}$. En el año 2018 se registró una cifra de 2,8 millones de niños y adolescentes con VIH, donde solo un $54 \%$ de ellos recibían tratamiento para el virus, frente a un $82 \%$ de mujeres embarazadas que padecían VIH y, en ese mismo año, se registraron alrededor de 360.000 nuevos casos en niños y adolescentes ${ }^{(6)}$. En el caso de Perú, el acumulado entre 2016 y 2020 contabilizó un total de 2.627 casos infectados con VIH, entre 0 y 19 años de $\operatorname{edad}^{(7)}$. 
Por su parte, es conocido que el padecer del VIH/SIDA genera ciertas limitantes para el sano desarrollo cognitivo y motor de los niños, más aún si éste no se somete al tratamiento antirretroviral. Tratamiento que ha disminuido la frecuencia y el grado de encefalopatías tempranas graves relacionadas al VIH, pero en los cuales las alteraciones cognitivas son significativas y comunes en la actualidad ${ }^{(8,9)}$. Entre estas alteraciones se pueden identificar un amplio rango de deficiencias neurológicas, como las visuales, motoras, de lenguaje y déficit cognitivo generalizado ${ }^{(10)}$.

La transmisión más frecuente del VIH a los infantes es durante el embarazo y la lactancia. En el primer caso se puede ver comprometido el neurodesarrollo con la sola exposición prenatal, cuando la activación inmunitaria materna afecta negativamente al cerebro fetal en desarrollo, entendiéndose neurodesarrollo como el proceso en el cual se adquieren habilidades madurativas en la nińez, y que está determinado en gran proporción por los aspectos biológicos y ambientales con los que estos se encuentren en constante interacción ${ }^{(10-}$ 12). De hecho, datos de diversos estudios demuestran que en los países de bajos ingresos, los niños nacidos en la pobreza tienen más probabilidades de experimentar deficiencias en el desarrollo cognitivo ${ }^{(12)}$.

Como respuesta a los factores biológicos y estímulos ambientales, se organiza el sistema nervioso como un sistema de relación que interactúa intrínsecamente, se desarrollan diferentes acciones como atención, intencionalidad, emoción, pensamiento, memoria, lenguaje, socialización y control motor, para actuar según las señales del medio ${ }^{(13)}$. Sin embargo, existe el riesgo de estar en contacto con ciertos factores ambientales que aumentan la aparición de problemas en el neurodesarrollo de los infantes, poniendo en riesgo aún más su salud, siendo generalmente observable este tipo de sucesos, en entornos de bajos ingresos y relacionado a la estabilidad psicológica de sus cuidadores, como pudiera presentarse en los casos donde la madre presenta alguna enfermedad ${ }^{(14)}$, como el VIH/SIDA.

El neurodesarrollo de los infantes es un proceso que se estudia desde el nacimiento hasta los 8 ańos de edad y en casos de patologías que potencialmente puedan incidir en el desarrollo neurológico, se reconocen 7 momentos luego del nacimiento, como los referentes para una intervención oportuna: al nacer, entre los 3 y 4 meses, 6 y 8 meses, 12 a 14 meses, 18 meses, 3 y 4 años, y 6 y 8 años de edad ${ }^{(15)}$, periodos en que la madre ejerce mayor influencia. En este sentido, en los factores de comportamiento asociados a la calidad del cuidado, como el estado psicológico de la madre, estaría la depresión pos parto y los factores de estrés asociados a la crianza, condiciones que se ha demostrado afectan tanto el adecuado crecimiento del niño, como a la relación madre e hijo ${ }^{(15,16)}$.

Son pocos los estudios acerca de la calidad del cuidado según la calidad del cuidador. Sin embargo, se reconoce que la persona objeto de cuidado ha de ser el centro de la atención del cuidador, debe recibir, de acuerdo a sus necesidades, cuidados que le permitan alcanzar su autonomía y bienestar. Por otro lado, el cuidador debe poseer habilidades comunicacionales, ser colaborador y asertivo, y asimismo, tener presente el estatus funcional, físico, mental y psicosocial de quien recibe los cuidados. Se trata de una estrecha relación en la que el cuidador se enfrenta a una carga de responsabilidad, desde un punto de vista objetivo, por las actividades en relación a las demandas del enfermo, pero subjetivo, ya que tendrá que lidiar con sus sentimientos, emociones y actitudes ${ }^{(17,18)}$, condiciones que determinan la calidad del cuidado, más si el cuidador pertenece al entorno familiar.

De lo expuesto, se entiende que el deterioro neurocognitivo en nińos pacientes con VIH/SIDA sería el resultado de un proceso multifactorial, donde ocurre la interacción de factores biológicos y del entorno, por tanto, los resultados neurocognitivos pueden variar de acuerdo al individuo, el ambiente en que estos se encuentren y los lugares en donde estos se desenvuelven, según el nivel socioeconómico y contexto cultural $^{(19)}$. Se ha observado que los nińos infectados por el VIH presentan más disfunciones neurológicas que los no infectados, a pesar de recibir tratamiento antirretroviral temprano, lo que no deja evidencia concluyente sobre el neurodesarrollo del infante con VIH/SIDA ${ }^{(19)}$.

Según lo anterior y como confirmación que el desarrollo neurológico es el resultado de múltiples factores, cabe preguntarse si parte del desarrollo neurológico, en estos casos, está influenciado por el tipo de apoyo y/o cuidado que recibe el infante, más allá del contexto socioeconómico donde se 
desenvuelve el menor.

Así, el objetivo de este estudio fue evaluar la calidad del cuidado familiar y su efecto en el neurodesarrollo de niños en edad preescolar y escolar, infectados con VIH/SIDA, hospitalizados en los servicios de Medicina Interna y Urgencias de 2 instituciones de salud, de la ciudad de Lima, Perú. De igual forma y con miras a implementar su uso en los protocolos de rutina, se evaluó la utilidad de los dos instrumentos aplicados en la investigación: Escala Abreviada de Desarrollo (EAD-3) del Ministerio de Salud de Colombia/ UNICEF $^{(20)}$ y el inventario Observaciones del Hogar para la Medición de Ambiente $(H O M E)^{(21)}$, para la valoración del neurodesarrollo y la calidad del cuidado de infantes con VIH/SIDA.

\section{MATERIAL Y MÉTODO}

Tipo de estudio y unidad de análisis: Bajo un enfoque cuantitativo se realizó un estudio tipo descriptivo de corte transversal, cuya unidad de análisis fue la diada paciente-cuidador/a, integrada por infantes de entre 3 y 6 años de edad, diagnosticados con VIH/SIDA, hospitalizados en los servicios de Medicina Interna y Urgencias de dos instituciones de salud de segundo nivel y el miembro familiar responsable. Los establecimientos seleccionados para el estudio están ubicados en el área metropolitana de la ciudad de Lima, Perú. Se trata de dos centros de atención que se consideran especializados en el tratamiento y la prevención de las poblaciones vulnerables que viven con el VIH/ SIDA y los niños en riesgo de infección.

Población y muestra: Debido al tamaño de la población en los servicios de Medicina Interna y Urgencias, y al hecho de que los diagnósticos de VIH en los niños se realizan de forma tardía porque los casos diagnosticados corresponden a madres sin diagnóstico y sin tratamiento antirretroviral a tiempo, se aplicó un muestreo intencional-censal, durante el periodo de junio a diciembre del año 2019, considerando que fue a partir del segundo trimestre del año que se obtuvo el consentimiento informado por escrito y la autorización de los centros hospitalarios. Así se conformaron 20 diadas paciente-cuidador con la totalidad de infantes hospitalizados y sus correspondientes cuidadores. El único criterio de inclusión fue que el representante legal, de forma voluntaria, aceptara participar en el estudio, al firmar un consentimiento informado.

Recolección de datos e instrumentos: El neurodesarrollo de los pacientes fue valorado mediante la Escala Abreviada de Desarrollo 3 (EAD-3), la cual mide las siguientes 4 dimensiones: motricidad gruesa, motricidad fino-adaptativa, audiciónlenguaje y personal-social. Cada una de ellas está estructurada con 30 ítems y clasificadas de acuerdo a la edad, ya que permite valorar el desarrollo desde el momento del nacimiento a término o tardío, hasta los 6 años. Para este estudio solo se aplicaron los ítems que corresponden al grupo etario a evaluar entre 3 y 6 años de edad que en el instrumento corresponden a los grupos o rangos numerados desde 9 a 12, a saber: 9) entre 36 meses y 1 día a 48 meses y 0 días; 10$)$ a partir de 48 meses y 1 día a 60 meses y 0 días; 11) entre 60 meses y 1 día a 72 meses y 0 días y 12) entre 72 meses y 1 día a 84 meses $^{(20)}$.

La valoración de la calidad de cuidado se midió durante la consulta externa del infante, considerando los datos sociodemográficos del cuidador, tales como edad, estado civil, grado de instrucción, procedencia, parentesco con el paciente, situación laboral, y tiempo de dedicación y de inicio del cuidado especial respecto al día del diagnóstico.

De igual forma, se valoraron las condiciones bajo las cuales el infante está siendo atendido en su hogar con el inventario Observaciones del Hogar para la Medición de Ambiente (Home Observation for Measurement of the Environment - HOME), diseñado originalmente por Caldwell y Bradley en 1984, para conocer los aspectos de la cantidad y calidad del apoyo social, emocional y cognitivo puesto a disposición del niño en el entorno del hogar, es decir, la calidad del entorno familiar en relación a los cuidados e interacciones estimulantes y de apoyo que reciben los infantes en el medio ambiente donde se desarrollan ${ }^{(21)}$.

De la escala $H O M E$ se han desarrollado muchas versiones de acuerdo a la edad de los nińos y para este estudio se aplicó una versión para primera infancia modificada en algunos de los ítems para adaptarla a la población a evaluar. Basándonos en la experiencia chilena, descrita por Bustos et al. ${ }^{(21)}$, manteniendo las características originales de puntuación a través de la observación y entrevista semiestructurada a 
los cuidadores, ya que es administrada en el hogar del infante. Se consideraron 45 ítems de respuestas dicotómicas SI/NO, diseñadas para valorar seis dimensiones: 1) receptividad; 2) aceptación de los padres; 3) organización del hogar; 4) materiales para el aprendizaje; 5) involucramiento de los padres; y 6) variedad en la estimulación. Al sumar las respuestas positivas se obtiene un puntaje final que, mientras más alto sea, indica mayor calidad del cuidado ${ }^{(20)}$.

En cuanto al neurodesarrollo, se aplicó un instrumento diseñado para hispanohablantes en Colombia, auspiciado por UNICEF entre 1987 y 1990. Aunque es poco conocido a nivel internacional, se utiliza principalmente para controlar y supervisar los programas de crecimiento y desarrollo con evaluaciones limitadas de su validez y está patrocinado por el Ministerio de Salud de Colombia ${ }^{(22)}$, pero recientemente algunos autores han informado de niveles adecuados de fiabilidad en la identificación de estados de neurodesarrollo y en la predicción del riesgo ante trastornos del desarrollo y de fuertes correlaciones con variables como el estado nutricional ${ }^{(23,24)}$, por tanto, se considera una importante herramienta de fácil y rápida aplicación para definir las estrategias de forma oportuna, que como acciones correctivas, permiten un adecuado desarrollo del menor en riesgo $^{(24)}$.

Control de calidad de los datos: La propiedad psicométrica de la fiabilidad del instrumento se obtuvo mediante el coeficiente alfa de Cronbach. La consistencia interna de la escala abreviada de desarrollo 3 (EAD-3) fue muy alta $(0,94)$, y el parámetro más representativo fue la Motricidad fina; lo mismo ocurrió con la escala $\operatorname{HOME}(0,93)$, y el parámetro más representativo fue la Aceptación de los padres.
Análisis de datos: Los datos obtenidos fueron procesados estadísticamente de forma descriptiva con la presentación de las frecuencias y porcentajes. En cuanto a la valoración de la relación entre ambas variables y establecer cuál de las dimensiones de la variable calidad del cuidado ejerce mayor influencia sobre la variable neurodesarrollo del infante, se aplicaron dos pruebas: el Coeficiente de correlación de Spearman (Rho) y la Regresión multivariada, basada en Modelos Lineales Generalizados Múltiples (MLGM), considerando un 95\% de confianza.

Estas pruebas se seleccionaron luego que se determinara el tipo de distribución de los datos, con la prueba de normalidad de los test de KolmogorovSmirnov y Shapiro-Wilk (Tabla1), teniendo mayor relevancia el test Shapiro-Wilk ya que por tratarse de una muestra menor a 50 , es el más recomendado ${ }^{(22)}$. Esta prueba arrojó (con $\mathrm{p}<0,05)$, que los datos no presentaban distribución normal, razón por la cual se utilizaron los test no paramétricos mencionados anteriormente. Los datos se procesaron en el programa SPSS ${ }^{\circ}$ versión 24.

Aspectos éticos: Para la realización de esta investigación se solicitó aprobación de la Dirección Central de las instituciones de salud y del comité de ética de la Facultad de Medicina Humana de la Universidad Nacional Mayor de San Marcos (Código de aprobación: 24034 de junio 2019). En correspondencia con la normativa de responsabilidad bioética, se cumplió con dar a conocer los objetivos del estudio a los representantes legales de los menores y sus cuidadores, quienes, al aceptar participar en el estudio, de forma voluntaria firmaron el consentimiento informado, el cual deja constar por escrito el compromiso del anonimato de las personas involucradas en el mismo.

Tabla 1. Tipo de distribución de los datos, de acuerdo a los estadísticos Kolmogorov-Smirnov y Shapiro-Wilk.

\begin{tabular}{lcccccc}
\hline \multirow{2}{*}{ Variables } & \multicolumn{3}{c}{ Kolmogorov-Smirnov* } & \multicolumn{3}{c}{ Shapiro-Wilk } \\
\cline { 2 - 7 } & Estadístico & gl & $\mathbf{p}$ & Estadístico & gl & p \\
\hline Calidad de cuidado &, 289 & 20 &, 000 &, 837 & 20 &, 000 \\
\hline Neurodesarrollo &, 256 & 20 &, 001 &, 818 & 20 &, 002 \\
\hline
\end{tabular}

* Corrección de significación de Lilliefors. 


\section{RESULTADOS}

Caracterización sociodemográfica de la diada paciente-cuidadora: De los 20 infantes que participaron en el estudio, la mayoría tenía entre 3 y 4 años, 30 y 35\% respectivamente. Asimismo, las personas responsables del cuidado, que en todos los casos se trató de mujeres entre 18 y 60 años de edad, el $40 \%$ tenía entre 36 y 59 años y el $35 \%$ entre 18 y 35 , representando con ello el grueso de la muestra. En el $70 \%$ se trataba de las propias madres de los menores. El 30\% de las cuidadoras era soltera y otro $30 \%$ era conviviente; tenían un nivel educativo mayoritariamente $(50 \%)$ de nivel secundario completo; el 35\% manifestó optar por trabajos remunerados de forma eventual y un 30\% tenía trabajo estable por horas; mayoritariamente, en un $70 \%$, señalaron que dedicaban las 24 horas del día al cuidado del menor (Tabla 2).

Tabla 2. Características sociodemográficas de las diadas infante con VIH/SIDA-cuidador familiar, junio a diciembre $2019(n=20)$.

\begin{tabular}{|c|c|c|c|c|}
\hline Integrantes de la diada & Variables sociodemográficas & & Fr & $\%$ \\
\hline \multirow[t]{4}{*}{ Infantes con VIH/SIDA } & Edad (años) & 3 & 6 & 30 \\
\hline & & 4 & 7 & 35 \\
\hline & & 5 & 4 & 20 \\
\hline & & 6 & 3 & 15 \\
\hline \multirow[t]{28}{*}{ Cuidadoras } & Edad (ańos) & $18-35$ & 7 & 35 \\
\hline & & $36-59$ & 8 & 40 \\
\hline & & $\geq 60$ años & 5 & 25 \\
\hline & Parentesco & Madre & 14 & 70 \\
\hline & & Hermana & 1 & 5 \\
\hline & & Abuela & 5 & 25 \\
\hline & Procedencia & Lima metropolitana & 6 & 30 \\
\hline & & Lima Sur & 2 & 10 \\
\hline & & Lima Este & 2 & 10 \\
\hline & & Lima Norte & 4 & 20 \\
\hline & & Provincia & 6 & 30 \\
\hline & Estado Civil & Soltera & 6 & 30 \\
\hline & & Conviviente & 6 & 30 \\
\hline & & Casada & 3 & 15 \\
\hline & & Separada & 2 & 10 \\
\hline & & Viuda & 3 & 15 \\
\hline & Grado de Instrucción & Primaria & 6 & 30 \\
\hline & & Secundaria & 10 & 50 \\
\hline & & Superior & 4 & 20 \\
\hline & Ocupación & Desempleada actualmente & 4 & 20 \\
\hline & & Con trabajo eventual & 7 & 35 \\
\hline & & Trabajo estable a tiempo completo & 3 & 15 \\
\hline & & Trabajo estable por horas & 6 & 30 \\
\hline & Tiempo (horas) dedicado al & 24 & 14 & 70 \\
\hline & cuidado & 7 a 23 & 5 & 25 \\
\hline & & $\leq 6$ & 1 & 5 \\
\hline & ¿Cuidado desde el momento del & SI & 19 & 95 \\
\hline & diagnóstico? & $\mathrm{NO}$ & 1 & 5 \\
\hline
\end{tabular}


Evaluación del neurodesarrollo del infante con VIH/SIDA: De acuerdo con el instrumento EAD3 , destaca que un $40 \%$ se encuentra en riesgo de padecer un problema en el desarrollo en las dimensiones de motricidad gruesa y personal-social respectivamente, mientras que bajo sospecha de presentar un problema de desarrollo, un $15 \%$ se focaliza en los componentes de motricidad fina y personal-social respectivamente (Tabla 3).

Calidad del cuidado y neurodesarrollo de los infantes: Se observó una asociación significativa, fuerte y positiva con un nivel de confianza del
95\% (Rho $=0,5252 ; \mathrm{p}=0,0221)$, que indica que mientras mayor sea el nivel de cuidado, en los ámbitos de atención y apoyo, mejor es, en general, el desarrollo neurológico del infante. Asimismo, cuando se valora de forma independiente cada una de las dimensiones que determinan el nivel de cuidado, de acuerdo al inventario HOME, son los ámbitos de Aceptación ( $\mathrm{R}=0,5220 ; \mathrm{p}=0,0229)$ y Materiales de aprendizaje $(\mathrm{R}=0,6026, \mathrm{p}=0,0086)$, los que presentan una fuerte y positiva influencia sobre el desarrollo neurológico (Tabla 4).

Tabla 3. Distribución porcentual del desarrollo neurológico según la Escala Abreviada del Desarrollo-3 en los infantes con diagnóstico VIH/SIDA, junio a diciembre 2019, n=20.

\begin{tabular}{lcccccccc}
\hline \multirow{2}{*}{ Diagnóstico } & \multicolumn{7}{c}{ Dimensiones del neurodesarrollo } \\
\cline { 2 - 9 } & \multicolumn{1}{c}{ Motricidad gruesa } & Motricidad fina & Audición/Lenguaje & Personal-social \\
\cline { 2 - 9 } & $\mathbf{n}$ & $\mathbf{0}$ & $\mathbf{n}$ & $\mathbf{\%}$ & $\mathbf{n}$ & $\mathbf{\%}$ & $\mathbf{n}$ & $\%$ \\
\hline $\begin{array}{l}\text { Desarrollo esperado para la } \\
\text { edad }\end{array}$ & 11 & 55 & 11 & 55 & 13 & 65 & 9 & 45 \\
\hline En Riesgo & 8 & 40 & 6 & 30 & 6 & 30 & 8 & 40 \\
\hline Con Sospecha & 1 & 5 & 3 & 15 & 1 & 5 & 3 & 15 \\
\hline
\end{tabular}

Tabla 4. Calidad de cuidados global y por dimensiones y el neurodesarrollo de infantes diagnosticados con VIH/ SIDA, junio a diciembre 2019, $\mathrm{n}=20$.

\begin{tabular}{|c|c|c|c|c|}
\hline \multicolumn{2}{|l|}{ Variables } & $\begin{array}{l}\text { coeficiente/ } \\
\text { sig. bilateral }\end{array}$ & $\begin{array}{l}\text { Calidad de } \\
\text { cuidados }\end{array}$ & Neurodesarrollo \\
\hline \multirow{2}{*}{\multicolumn{2}{|c|}{ Calidad de cuidados (global) }} & rho & 1,000 &, 5252 \\
\hline & & $\mathrm{p}$ & & 0,0221 \\
\hline \multirow{12}{*}{$\begin{array}{l}\text { Dimensiones de la Calidad de } \\
\text { cuidado }\end{array}$} & \multirow[t]{2}{*}{ Responsividad } & rho & & , 194 \\
\hline & & $\mathrm{p}$ & & 0,3978 \\
\hline & \multirow[t]{2}{*}{ Aceptación } & rho & &, 5220 \\
\hline & & $\mathrm{p}$ & & 0,0229 \\
\hline & \multirow[t]{2}{*}{ Organización } & rho & &, 4215 \\
\hline & & $\mathrm{p}$ & & 0,0662 \\
\hline & \multirow[t]{2}{*}{ Materiales de aprendizaje } & rho & & ,6026 \\
\hline & & $\mathrm{p}$ & & 0,0086 \\
\hline & \multirow[t]{2}{*}{ Involucramiento } & rho & & ,2736 \\
\hline & & $\mathrm{p}$ & & 0,2330 \\
\hline & \multirow[t]{2}{*}{ Variedad } & rho & &, 0039 \\
\hline & & $\mathrm{p}$ & & 0,9863 \\
\hline
\end{tabular}


Para corroborar la influencia de las dimensiones del inventario HOME, sobre el neurodesarrollo de los infantes según EAD-3, se realizó un análisis de regresión multivariado por MLGM, el cual arrojó similar resultado (Tabla 5). Con $\mathrm{p}=0,0395$ se obtuvo un coeficiente de determinación $\mathrm{R}^{2}=0,5919$, que indica que la calidad del cuidado de acuerdo a $H O M E$, solo predice el 59,19\% de la variabilidad del neurodesarrollo, denotando que no todas las dimensiones influyen sobre dicha variable. El análisis de varianza de la contribución de cada una de las dimensiones al MLGM demuestra que las únicas dimensiones del inventario $H O M E$ que influyen de manera significativa sobre el neurodesarrollo son Aceptación $(\mathrm{p}=0,0332)$ y Materiales de aprendizaje con $\mathrm{p}=0,0466$, observándose que el efecto de la calidad de la atención sobre el neurodesarrollo de los infantes se evaluó con coeficientes de correlación positivos para todos los parámetros evaluados, pero no todos fueron estadísticamente significativos, como se muestra en la Tabla 5, puesto que sólo la Aceptabilidad y los Materiales de aprendizaje se obtuvieron por debajo de 0,05 y en ambos casos se obtuvo una correlación positiva moderada.

Tabla 5. Calidad de cuidado y Neurodesarrollo en infantes diagnosticados con VIH/SIDA, junio a diciembre 2019, $\mathrm{n}=20$.

\begin{tabular}{lrrrrr}
\hline \multicolumn{7}{l}{ Análisis de Varianza para Neurodesarrollo y Calidad de Cuidado Familiar } \\
\hline Fuente & Suma de Cuadrados & Gl & Cuadrado Medio & Razón-F & p \\
\hline Modelo & 72,9214 & 6 & 12,1536 & 3,14 & 0,0395 \\
Residuo & 50,2786 & 13 & 3,86758 & & \\
Total (Corr.) & 123,2 & 19 & & & \\
\hline Análisis de varianza por cada dimensión individual & & & & \\
\hline Fuente & Suma de Cuadrados & Gl & Cuadrado Medio & Razón-F & p \\
\hline Responsividad & 0,92748 & 1 & 0,92748 & 0,24 & 0,6325 \\
Aceptación & 21,9385 & 1 & 21,9385 & 5,67 & 0,0332 \\
Organización & 5,00366 & 1 & 5,00366 & 1,29 & 0,2759 \\
Materiales de aprendizaje & 18,6985 & 1 & 18,6985 & 4,83 & 0,0466 \\
Involucramiento & 4,88571 & 1 & 4,88571 & 1,26 & 0,2814 \\
Variedad & 11,2995 & 1 & 11,2995 & 2,92 & 0,1111 \\
Residuo & 50,2786 & 13 & 3,86758 & & \\
Total (corregido) & 123,2 & 19 & & & \\
\hline
\end{tabular}

\section{DISCUSIÓN}

En la investigación se evaluó el efecto de la calidad de los cuidados del familiar en el neurodesarrollo del infante, en su mayoría entre 3 y 6 años diagnosticados con VIH/SIDA, hospitalizados en los servicios de Medicina Interna y Urgencias de dos hospitales de Lima durante el 2019. Para ello, se utilizaron herramientas reconocidas y previamente validadas en el Perú para el grupo etario en estudio, como la Escala Abreviada de Desarrollo - 3 (EAD3) para evaluar el neurodesarrollo de los infantes y el Home Observation for Measurement of the Environment - HOME para evaluar la calidad de cuidado del familiar hacia el niño.

Los infantes diagnosticados con VIH/SIDA participantes del estudio, en un $65 \%$, tenían entre 3 y 4 años y al 70\% lo cuidaban sus propias madres, quienes en un $40 \%$ tenían entre 36 y 59 ańos. Estas observaciones coinciden con diversos estudios, los cuales han evidenciado que la mayoría de las personas que asumen el rol de cuidadores son: mujeres adultas, en pleno desarrollo de su productividad, realizan las tareas propias de su rol como mujer, dado que el $100 \%$ de los cuidadores son de género femenino y que, además, el 30\% son solteras y otro $30 \%$ son convivientes. Por otra parte, el parentesco que guardan con el infante, 
en su mayoría (70\%), es el de madre, mientras que el $25 \%$ es el de abuela, lo cual guarda sentido con el grupo etario ${ }^{(4,23-26)}$. En relación al grado de instrucción, llamó la atención que, en este estudio, la mayoría tiene secundaria, mientras que en otros con similar nivel socioeconómico, los cuidadores habían alcanzado solo el nivel primario, situación que posiblemente puede afectar el ejercicio del cuidado, especialmente en la estimulación para el desarrollo cognoscitivo ${ }^{(4,23,27)}$.

Por otro lado, el estudio buscó evaluar el retraso en el desarrollo neurológico de los infantes considerando para ello la tercera versión del instrumento pues su rango de evaluación es hasta los 7 años. Se observó, inicialmente, que se trata de una escala que permite realizar un diagnóstico de riesgo en el desarrollo motor, del lenguaje y de las relaciones con los demás, como Nivel de interacción social y Expresión de emociones y Aprendizajes en el autocuidado ${ }^{(20,23)}$, con la que se determinó que el 30 y $40 \%$ de los nińos presenta un nivel alto de riesgo en todas las dimensiones que se evalúan, es decir, presentan retraso en las Habilidades motoras, del Habla y en lo Personal-social, correspondiente para su edad.

Se trata de un número importante, que indica que el estado de salud, que caracteriza el diagnóstico VIH/SIDA, también afecta a su desarrollo neurológico. Similares resultados reportan Familiar et al. ${ }^{(10)}$ y Rie et al. ${ }^{(28)}$, quienes evalúan el desarrollo motor y cognitivo en niños con VIH, que, si bien emplean otros instrumentos, observan comparativamente con niños sanos, que hay retraso en las habilidades motoras y cognitivas con respecto a la edad, en aquellos que presentan la infección viral.

Ahora bien, en este estudio valoramos a la par el neurodesarrollo y la calidad de cuidado empleando el inventario $H O M E$, el cual ha sido útil en la valoración del ambiente familiar con respecto a las condiciones socioeconómicas o nutricionales, desde el punto de vista del adecuado desarrollo de los infantes, a nivel antropométrico o índice de masa corporal (IMC) ${ }^{(29,30)}$, determinándose que en el neurodesarrollo influyen diversos factores, entre los que se pueden mencionar los socioeconómicos y ambientales.

En este estudio, la calidad de cuidado presentó una fuerte asociación, estadísticamente significativa, con el neurodesarrollo medido con EAD-3, donde específicamente las dimensiones de Aceptación y Medios de aprendizaje, de acuerdo a los estadísticos aplicados, son las áreas que más afectan el desarrollo infantil.

Esta correlación puede indicar que quizás por la baja capacidad de aceptación de la enfermedad y las pocas herramientas de aprendizaje que tiene el cuidador, en parte por su nivel educativo, interfieren en una adecuada calidad de los cuidados del infante y en su adecuada estimulación para su desarrollo. Por supuesto, la calidad de cuidado apenas es responsable de un $59 \%$ de la predicción del rezago del neurodesarrollo, lo que permite reconocer la presencia de otros factores que pueden estar interviniendo en el mismo, como la situación económica y el estado nutricional, entre otros. Esto relacionado a las características específicas de los infantes y de sus cuidadores participantes de este estudio, quienes son susceptibles a su vez de presentar cargas de estrés ya que las madres también presentaban diagnóstico VIH/SIDA, lo que puede condicionar estados de ánimo que igualmente interfieran en la calidad de atención, como en este caso. Resultado similar reportaron Familiar et al. ${ }^{(10)}$ al contrastar el inventario HOME y el neurodesarrollo de infantes con VIH, quienes además de observar un retraso del desarrollo cognitivo, apreciaron una relación directa de este con las puntuaciones del inventario.

Conocer las condiciones en que se lleva a cabo el cuidado familiar de un infante debe servir de base para que el trabajo que realice el personal de enfermería en las instituciones de salud los beneficie realmente. A partir de este conocimiento se pueden generar programas de orientación y adiestramiento sistemático de aplicación de estrategias para que los cuidadores familiares puedan contribuir al cuidado de sus niños enfermos en el hogar, enriquecer sus conocimientos y habilidades para favorecer la estimulación temprana y por tanto el desarrollo neurológico de los niños ${ }^{(10,31)}$.

Los datos presentados en este estudio son preliminares, no definitivos, por tanto, deben motivar más investigaciones en el área, que contribuyan a la validación de los instrumentos utilizados, como pruebas con mayor número de muestras o también contrastando con diversas condiciones del entorno o de la salud de los infantes, a fin de determinar si efectivamente puede ser recomendado en el país, para su uso rutinario 
en la valoración tanto del nivel de neurodesarrollo como del cuidado familiar del infante.

Incorporar limitaciones que tuvo el estudio y cómo fueron subsanadas.

\section{CONCLUSIÓN}

El diagnóstico de VIH/SIDA en los infantes participantes del estudio afecta su neurodesarrollo, ocasionando un retardo en el desenvolvimiento de las habilidades a nivel motor, cognitivo y en la interrelación con otros y expresión de sus propias emociones.

Asimismo, la calidad del cuidado del familiar hacia el niño ejerce un efecto positivo en el neurodesarrollo de los infantes, por lo que se considera necesario fortalecer los diferentes medios de apoyo del cuidador familiar, para disminuir el estrés o sobrecarga relacionada con su tarea.

La Escala Abreviada de Desarrollo (EAD-3) se presenta como una herramienta de gran utilidad, que permite de forma fácil, rápida y no invasiva, tener un diagnóstico de riesgo, que permite tomar medidas oportunas para corregir y procurar un avance significativo en la mejora de la calidad de vida infantil.

Las investigaciones futuras podrían estudiar el impacto del apoyo social percibido por el cuidador en la calidad de los cuidados, la depresión inducida por el estrés del cuidador, el estado de salud de un familiar de un paciente enfermo, ya que su conocimiento, así como su estado de salud pueden depender del nivel de estimulación y amor que el infante necesita para un adecuado desarrollo del sistema nervioso.

\section{REFERENCIAS}

1. Black M, Walker S, Fernald L, Andersen C, DiGirolamo A, Lu Ch. et al. Early childhood development coming of age: science through the life course. Lancet [Internet]. 2017 [citado 20 mar 2020]; 89: 77-90. Disponible en: https://doi. org/10.1016/S0140-6736(16)31389-7

2. Rodríguez LM, Monge VS. La desnutrición y el estrés van a la escuela: pobreza infantil y neurodesarrollo en América Latina. Innovaciones educativas [Internet]. 2017 [citado $20 \mathrm{mar}$ 2020]; 19(27): 55-70. https://doi.org/10.22458/ ie.v19i27.1955

3. Grantham-McGregor S. What is the best design for early childhood interventions? Dev Med Child Neurol [Internet]. 2016 [citado 20 mar 2020]; 58: 222-223. Disponible en: https://onlinelibrary. wiley.com/doi/full/10.1111/dmcn.12951

4. Boivin M, Nakasujja N, Familiar-Lopez I, Murray S, Sikorskii A, Awadu J, et al. Effect of Caregiver Training on the Neurodevelopment of HIV-Exposed Uninfected Children and Caregiver Mental Health: A Ugandan Cluster-Randomized Controlled Trial. J Dev Behav Pediatr [Internet]. 2017 [citado 20 mar 2020]; 38(9): 753-764. Disponible en: https:// doi.org/10.1097/dbp.0000000000000510

5. Sugandhi N, Rodrigues J, Kim M, Ahmed S, Amzel A, Amzel A, et al. HIV Exposed Infants: Rethinking care for a lifelong condition. AIDS [Internet]. 2013 [citado 20 mar 2020]; 27(2): 187-195. Disponible en: https://doi.org/10.1097/ qad.0000000000000090

6. UNICEF. VIH y SIDA, UNICEF aspira a una generación sin SIDA: un futuro en el que todos los niños y sus familias estén protegidos de la infección por el VIH y vivan libres del SIDA [Internet]. Programa versión español [citado 21 mar 2020]. Disponible en: https://www.unicef.org/es/vih

7. Ministerio de Salud. Situación epidemiológica del VIH-Sida en el Perú. Boletín VIH. Centro Nacional de Epidemiología, Prevención y Control de Enfermedades [Internet]. 2020 [citado $26 \mathrm{feb}$ 2021]. Disponible en: https://www.dge.gob.pe/ portal/docs/vigilancia/vih/Boletin_2020/febrero. pdf

8. Brahmbhatt H, Boivin M, Ssempijja V, Kigozi G, Kagaayi J, Serwadda D, et al. Neurodevelopmental benefits of antiretroviral therapy in Ugandan children aged 0-6 years with HIV. J Acquir Immune Defic Syndr [Internet]. 2014 [citado 21 mar 2020]; 67: 316-322. Disponible en: https:// www.ncbi.nlm.nih.gov/pubmed/25314252

9. Boivin M, Barlow-Mosha L, Chernoff M, Laughton B, Zimmer B, Joyce C, et al. Neuropsychological performance in African children with HIV enrolled in a multisite antiretroviral clinical trial. AIDS [Internet]. 2018 [citado 21 mar 2020]; 32: 189204. Disponible en: https://www.ncbi.nlm.nih. gov/pubmed/29112069

10. Familiar I, Collins S, Sikorskii A, RuisenorEscudero H, Natamba B, Bangirana $P$, et al. Quality of caregiving is positively associated with neurodevelopment during the first year of life among HIV-exposed uninfected children in Uganda. J Acquir Immune Defic Syndr [Internet]. 2018 [citado 21 mar 2020]; 77(3): 235-242. Disponible en: https://www.ncbi.nlm.nih.gov/ 
pubmed/29210832

11. Sirois P, Huo Y, Williams P, Malee K, Garvie PA, Kammerer B, et al. Safety of perinatal exposure to antiretroviral medications: developmental outcomes in infants. Pediatr Infect Dis J [Internet]. 2013 [citado 21 mar 2020]; 32(6): 648-55. Disponible en: https://www.ncbi.nlm.nih.gov/pmc/arti cles/PMC3723344/

12. Walker S, Wachs T, Grantham-McGregor S, Black $\mathrm{M}, \mathrm{Nel}$ son Ch, Huffman S, et al. Inequality in early childhood: risk and protective factors for early child development. Lancet [Internet]. 2011 [citado 22 mar 2020]; 378(9799): 1325-38. Disponible en: https://doi.org/10.1016/s0140-6736(11)60555-2

13. Viquillon RY, Díaz LY, López GY, González ME. Módulo neurofisiología en el sistema informático "sistema de evaluación del neurodesarrollo en nińos" para el hospital pediátrico William Soler [Internet]. 2013 [citado 22 mar 2020]. Disponible en: http://www.jornada2014.sld.cu/index.php/jor nada/2014/paper/download/20/42

14. Surkan P, Kennedy C, Hurley K, Black M. Maternal depression and early childhood growth in developing countries: systematic review and metaanalysis. Bull World Health Organ [Internet]. 2011 [citado 22 mar 2020]; 89(8): 608-15. Disponible en: https://www.who.int/bulletin/volumes/ 89/8/11-088187/en/

15. Domínguez DF. Neurodesarrollo y estimulación temprana. Rev Cubana Pediatr [Internet]. 2019 [citado 22 mar 2020]; 91(2): e819. Disponible en: http://scielo.sld.cu/scielo.php?script=sci_arttext \& pid $=$ S0034-75312019000200001\&lng=es

16. Cooper P, Tomlinson M, Swartz L, Woolgar M., Murray L, Molteno C. Post-partum depression and the mother-infant relationship in a South African peri-urban settlement. The British Journal of Psychiatry [Internet]. 1999 [citado 22 mar 2020]; 175(6): 554-558. Disponible en: https://doi. org/10.1192/bjp.175.6.554

17. Arizmendi MDZ. La calidad del cuidado y la satisfacción de la persona. Enfermería Universitaria [Internet]. 2007 [citado 26 feb 2021]; 4(2): 3438. Disponible en: https://doi.org/10.22201/eneo. 23958421e.2007.2.461

18. Crespo M, Rivas M. La evaluación de la carga del cuidador: una revisión más allá de la escala de Zarit. Clínica y salud [Internet]. 2015 [citado 26 feb 2021]; 26(1): 9-15. Disponible en: https://doi. org/10.1016/j.clysa.2014.07.002

19. Debeaudrap P, Bodeau-Livinec F, Pasquier E, Germanaud D, Tetang NS, Nlend AN, et al. Neurodevelopmental outcomes in HIV-infected and uninfected African children. AIDS [Internet]. 2018 [citado 23 mar 2020]; 32(18): 2749-2757.
Disponible en: https://www.ncbi.nlm.nih.gov/pub $\mathrm{med} / 30289800$

20. Moreno S, Ganados C, Rodríguez N, Gómez C. Escala Abreviada de Desarrollo - 3. Bogotá: Editorial del Ministerio de Salud de Colombia [Internet]. 2016 [citado 27 feb 2021]. Disponible en: https://www.minsalud.gov.co/sites/rid/Lists/Bi bliotecaDigital/RIDE/VS/PP/ENT/Escala-abrevia da-de-desarrollo-3.pdf

21. Bustos CC, Herrera MO, Mathiesen ME. Calidad del ambiente del hogar: inventario HOME como un instrumento de medición. Estudios pedagógicos [Internet]. 2001 [citado 27 feb 2021]; (27): 7-22. Disponible en: http://dx.doi.org/10.4067/S071807052001000100001

22. Castro VJ, Cobos RCR. Análisis de escalas para la evaluación del desarrollo infantil usadas en América: Una revisión de literatura. Movimiento Científico [Internet]. 2018 [citado 02 mar 2021]; 10(2): 7282. Disponible en: https://revmovimientocientifico. ibero.edu.co/issue/view/101

23. Bustos LGM, Retamal-Matus HF, Amador RE, Ramos LS, Coronel ChE, Martínez FV, et al. Relación entre el estado nutricional y desarrollo motor en niños de 0 a 5 años de la etnia indígena Kankuama. Nutr Clín Diet Hosp [Internet]. 2018 [citado 02 mar 2021]; 38(4): 110-115. Disponible en: http://dx.doi.org/10.12873/384bustos

24. Jojoa LFR, Mayor YS, Pino VHC, Torres JDL. Rezagos del crecimiento y desarrollo en niños entre 18-60 meses en un hogar infantil de Palmira 2018 B. En XVIII Congreso de la Sociedad Cubana de Enfermería [Internet]. 2019 [citado 02 mar 2021]. Disponible en: http://enfermeria2019.sld.cu/index. php/enfermeria/2019/paper/viewFile/631/269

25. Droppelmann G. Pruebas de normalidad. Rev Actuali Clínc Meds [Internet]. 2018 [citado 23 mar 2020]; 2(1): 39-43. Disponible en: https://www. meds.cl/wp-content/uploads/Art-5.-GuillermoDroppelmann.pdf

26. Campos MS, Durán EY, Rivera R, Páez N, Carrillo G. Sobrecarga y apoyos en el cuidador familiar de pacientes con enfermedad crónica. Rev Cuid [Internet]. 2019 [citado 24 mar 2020]; 10(3): e649. Disponible en: http://dx.doi.org/10.15649/ cuidarte.v10i3.649

27. Ruiz LF, Gordillo AM, Galvis CR. Factores condicionantes básicos en cuidadores informales de pacientes crónicos en el domicilio. Rev Cuid [Internet]. 2019 [citado 24 mar 2020]; 10(3): e608. Disponible en: http://dx.doi.org/10.15649/ cuidarte.v10i3.608

28. Rie A, Dow A, Mupuala A, Stewar P. Neurodevelopmental Trajectory of HIV-Infected Children Accessing Care in Kinshasa, Democratic 
Republic of Congo. Epidemiology and Social Science. J Acquir Immune Defic Syndr [Internet]. 2009 [citado 25 mar 2020]; 52(5): 636-642. Disponible en: https://doi.org/10.1097/QAI.0b013e3 $181 \mathrm{~b} 32646$

29. Rivera GR, Figueroa OM, Soler LKM, Sánchez C, Ávila RH. Experiencia de la aplicación y criterios para la interpretación de dos versiones del Inventario HOME para infantes de 0 a 3 años de vida. Salud mental [Internet]. 2010 [citado 02 mar 2021]; 33(1): 57-66. Disponible en: http://www.scielo.org.mx/scielo.php?script=sci_ arttext\&pid=S0185-33252010000100007

30. López BF, Cubides MM, Garibotto G, Berón, C, Sorio R. Medición de la calidad del entorno familiar de los niños pequeños en Uruguay: Gra- diente socioeconómico en el inventario HOME. Nota Técnica del Banco Interamericano de Desarrollo [Internet]. 2018 [citado 02 mar 2021]; IDB TN 1550. Disponible en: https://publications. iadb.org/publications/spanish/document/ Medici\%C3\%B3n_de_la_calidad_del_ entorno_familiar_de_los_ni\%C3\%B 1 os_ peque $\%$ C3\%B1os_en_Uruguay_Gradientes_ socioecon\%C3\%B3micos_en_el_inventario_ HOME_es_es.pdf

31. Díaz-Morales K, Reyes-Arvizu J, Morgado-Nájera $\mathrm{K}$, Everardo-Domínguez D. Síntomas en niños con cáncer y estrategias de cuidado familiar. Rev Cuid [Internet]. 2019 [citado 22 mar 2020]; 10(1): e597. Disponible en: http://dx.doi.org/10.15649/ cuidarte.v10i1.59 